\title{
Who gets admitted to the Chest Pain Unit (CPU) and how do we manage them? Improving the use of the CPU in Waikato DHB, New Zealand
}

\author{
Judith Jade, Paul Huggan, Douglas Stephenson \\ Waikato DHB, New Zealand
}

\begin{abstract}
Chest pain is a commonly encountered presentation in the emergency department (ED). The chest pain unit at Waikato DHB is designed for patients with likely stable angina, who are at low risk of acute coronary syndrome (ACS), with a normal ECG and Troponin T, who have a history which is highly suggestive of coronary artery disease (CAD). Two issues were identified with patient care on the unit (1) the number of inappropriate admissions and (2) the number of inappropriate exercise tolerance tests.
\end{abstract}

A baseline study showed that $73 \%$ of admissions did not fulfil the criteria and the majority of patients $(72 \%)$ had an exercise tolerance test (ETT) irrespective of clinical picture. We delivered educational presentations to key stakeholders and the implementation of a new fast track chest pain pathway for discharging patients directly from the ED. There was an improvement in the number of patients inappropriately admitted, which fell to $61 \%$. However, the number of inappropriate ETTs did not decrease, and were still performed on $76.9 \%$ of patients.

\section{Problem}

The chest pain unit (CPU) at Waikato DHB, Hamilton, New Zealand is a four bedded unit designed for patients with an episode of chest pain thought to be secondary to ischemic coronary artery disease but without features of acute coronary syndrome (ACS). Access to CPU is via the emergency department (ED) doctors who can admit patients directly or via the cardiology registrar.

The chest pain unit admission guidelines are as follows: at least two out of three features of angina (central chest pain, brought on by exertion, relieved by rest or GTN), with a negative Troponin T and no ischemic ECG changes. The pain should have resolved, should be the patient's only problem, and should appear to have a cardiac cause.

Despite clear guidelines, a large number of patients admitted to CPU do not fulfil the criteria. While working on CPU at Waikato it became clear that patients were frequently admitted with nonanginal chest pain; for example, with pleuritic pain caused by a chest infection or tenderness from costochondritis, creating unnecessary hospital admissions and expense. Occasionally patients were admitted with other medical problems or complex cardiological histories and would be more appropriately managed on a medical or cardiology inpatient ward.

From observing practice on CPU, all patients admitted seemed to have exercise tolerance testing, despite their presentation, creating high false positive rates. This led us to question how we could improve the risk stratification process and improve the use of ETTs.

\section{Background}

Chest pain is a very common presentation, accounting for up to
$25 \%$ of emergency hospital admissions.[1] There are multiple causes of chest pain and identifying patients with coronary artery disease $(C A D)$ relies on careful clinical history taking. Typical anginal chest pain is defined as central chest pain, brought on by exertion and relieved by rest or glyceryl trinitrate (GTN) spray.[1] If a patient has two out of three features this is considered atypical angina and if one or less than this is non-anginal chest pain. A diagnosis of angina should be excluded if the pain is non-anginal.[1]

Chest pain units can help identify which patients have occlusive coronary artery disease that possibly needs percutaneous intervention, and help optimise medical management in patients that have anginal symptoms without features of acute coronary syndrome (ACS). This reduces cost compared to admission to a coronary care unit [2] and can safely reduce length of stay.[3]

Exercise tolerance testing is often used to identify patients with possible occlusive coronary artery disease that are not known to have CAD, or to help make treatment decisions in patients with CAD. However, this test has inherent problems in that it has a low sensitivity (68\%) and specificity ( $77 \%)$, leading to false negative and false positive results that can confuse management and lead to unnecessary and sometimes costly further investigations.

Probabilistic reasoning should be used to help decide when to use exercise testing.[4] If low risk patients are exercise tested, positive results are likely to be false positives and negative tests would not change the patient's management. NICE guidelines propose risk stratifying patients based on chest pain presentation, age, and CAD risk factors, and only using ETTs as part of the work up in the intermediate and high risk groups.[1] Those with typical angina do not necessarily need further investigation.[1]

It is important that patients are not incorrectly sent home with acute coronary syndrome; some studies report that this occurs in $2.1 \%$ of chest pain attendances.[2] This most often occurs if ECG changes 
are missed. However, research has shown that the majority of patients with chest pain that has resolved, with no ECG changes and a normal Troponin T level, can be safely discharged direct from ED without the requirement for admission. In a large study by Brandstein et al, an undetecatble Troponin $\mathrm{T}$ and no ischemic changes on ECG had a negative predictive value for myocardial infarction in 30 days of $99.8 \%$ and of death within 30 days of $100 \%$.[5] Than et al showed that instituting an accelerated chest pain pathway within ED in Christchurch Hospital, New Zealand, allowed five out of six patients to be discharged within six hours.[6] Rathod et al showed that chest pain pathways can improve the quality of referrals to chest pain units by improving discrimination between cardiac and non-cardiac chest pain.[7] Other quality improvement projects have shown reductions in admissions through the institution of chest pain pathways, based largely on clinical assessment, including accurate history taking.[8]

\section{Baseline measurement}

For this project various measures were considered, in the three distinct domains of quality: structure, process, and outcome.

Our structure measure was (1) speciality referring to CPU (eg, general medicine or cardiology).

Our process measures were three-fold: (1) Number of patients admitted inappropriately to CPU (2) Number of patients admitted to CPU with non-anginal chest pain (3) Number and characteristics of patients who were referred for exercise tolerance test (ETT) from CPU.

Our outcome measure was: (1) Number of patients diagnosed with occlusive coronary artery disease from admission to CPU.

There were 45 patients admitted to the chest pain unit during a one month period in May 2014; 59\% were male and $41 \%$ female, with an average age of 55 years old. One patient was excluded from the audit as their notes were unavailable. Baseline measurement clearly illustrated the problem we had identified. Seventy three percent (32) of patients did not fulfil the admissions criteria for CPU and $55 \%(24)$ had non-angina chest pain. Cardiology had referred or were consulted on 21 out of the 44 patients audited (47.7\%). Fourteen percent of these patients had non-anginal chest pain (66.6\% of cardiology admissions). Despite this, exercise tolerance tests were performed on $72 \%$ (31) of all admissions to CPU and $55 \%$ (17) of ETTs were done on patients with non-anginal chest pain. The majority of patients that had positive ETTs had in fact presented with non-anginal chest pain. None of the patients with non-anginal chest pain were diagnosed with occlusive coronary artery disease on discharge, and hence all these results can be considered false positives. Patients admitted with non-anginal chest pain did not have their management altered by admission to the CPU, even if they had a positive ETT. There were no diagnoses of occlusive coronary artery disease made.

\section{Design}

The patient journey from attendance to hospital with chest pain to discharge can be complex. This pathway needed to be simplified and a risk stratification approach needed to be taken; so that high risk patients are correctly identified, and patients with non-anginal chest pain fit for discharge avoid an unnecessary hospital stay.

The intervention was two fold. The initial part of the intervention was educational, based on our baseline data, about the utility of exercise testing (ETT) in patients with chest pain. This was in the form of power-point audit presentations, delivered to the emergency department and general medicine, with an emphasis on risk stratification. Through the use of case study examples from the audit and Fagan nomograms we highlighted the need for risk stratification before ordering an ETT.

The second part of the intervention was the development of a pathway to increase discharge of low risk patients from the emergency department and improve the appropriateness of patients admitted to the chest pain unit.

\section{Strategy}

PDSA cycle 1 involved planning the measurements to be taken and gathering all the data for patients admitted to CPU for a month long period. The data were then collated and we used this to create the educational power-point presentations for ED and general medical staff presentations. This put the issue on the agenda of both departments and started influencing doctors behaviour in terms of admission practices and highlighted the need to risk stratify.

PDSA cycle 2 involved consultant led meetings between ED, general medicine, and cardiology which led to the initiation of a new fast track chest pathway, with the aim of discharging more patients with non-anginal chest pain at low risk, directly from the emergency department. Education was given by senior ED staff to juniors about the aims and use of the new pathway. The pathway is outlined in figure 1. Practice was then re-audited.

See supplementary file: ds4610.docx - "Figure 1 Fast track chest pain pathway"

\section{Post-measurement}

After the second PDSA cycle we re-audited our practice on the CPU, taking a random sample of 13 patients during a two week period in November 2014. Seven of the 13 patients were referred to CPU by cardiology, the others were referred by ED. Out of the 13 patients admitted only five fulfilled the admissions criteria, $61.5 \%$ were inappropriate admissions. 53.8\% (seven) had non-anginal chest pain. Exercise tolerance tests were performed on $76.9 \%$ of patients. $50 \%$ of the ETTs done were on patients with non-anginal chest pain. Twelve out of the 13 patients were discharged without angiogram, further investigations or follow up. Only one patient, who had typical angina, was investigated further with an out-patient nuclear perfusion scan and cardiology follow up. No angiograms were performed or planned and no occlusive coronary disease was identified. 
Interestingly, there was a difference in the characteristics of the patients admitted by the two specialties. Cardiology had been consulted on seven of the 13 cases and had admitted six out of the seven patients with non-anginal chest pain. In contrast, there was a marked improvement in the appropriateness of referrals from ED, only admitting one patient with non-anginal chest pain.

\section{Lessons and limitations}

A number of valuable lessons were learnt whilst undertaking this project; initially the problem was identified but clear project aims were not developed. This led to collecting an excess of data, thought to be possibly useful at a later stage. In future quality improvement projects it will be more effective and efficient to collect smaller amounts of more relevant data from the outset. This will save time, which could be dedicated to focusing on implementing the changes which ultimately improve care.

This project underlined the importance of multiple small PDSA cycles to monitor progress. The time spent collecting irrelevant baseline data could have been reallocated to more numerous small test cycles, which would result in a better intervention.

It became evident in re-auditing our practice that we had not identified all the key stakeholders in this process, as the ED referrals improved but there was no real change in the cardiology referrals. The cardiology registrars are often the gatekeeper to admissions to CPU out of hours, so could have offered key insights and influence. In future quality improvement projects it will be important to identify all key stakeholders at the outset.

\section{Conclusion}

There were two parts to the problem identified; inappropriate admissions to CPU and the inappropriate use of ETTs. An underlying theme in both these problems is a culture of anxiety in the medical profession about missing patients who in fact have acute coronary syndrome. There are a multitude of articles in the literature that describe successfully and safely discharging patients who have had chest pain early.[3,5,6] The key is in taking an accurate clinical history and being able to risk stratify patients.[7] Our work has provided a learning tool and talking point about the use of risk stratification, and with the use of the fast track pathway doctors can feel more confident about discharging patients from ED. Changes in medical culture take a long time and may be difficult to quantify.

Through our intervention we saw some improvement in the admissions to $\mathrm{CPU}$, particularly in the appropriateness of referrals from ED and further work is needed to continue this. There was no change in the appropriateness of ETTs in assessing patients with chest pain, and greater improvement is needed.

No difference was seen in the appropriateness of cardiology referrals to CPU during this process. Greater success may have been achieved if the cardiology registrar had been identified as a key stakeholder at the start of this process. Future work on this quality improvement project should involve all stakeholders to strive for further change.

\section{References}

1. Cooper A, Timmis A, Skinner J. Assessment of recent onset chest pain or discomfort of suspected cardiac origin: summary of NICE guidance. BMJ 2010;340:c1118.

2. Lee TH, Goldman L. Evaluation of the patient with acute chest pain. N Engl J Med 2000;342:1187-95

3. Wiese, M. Reducing short-stay hospital admissions by ruling out non-ST elevation myocardial infarction and estimating coronary artery disease likelihood on an emergency department observation ward. BMJ Qual Improv Report 2013;2: doi:10.1136/bmjquality.u201080.w695

4. Doust J. Diagnosis in General Practice. Using probabilistic reasoning. BMJ 2009;339:b3823.

5. Bandstein N, Ljung R, Johansson M, et al. Undetectable high-sensitivity cardiac troponin $\mathrm{T}$ level in the emergency department and risk of myocardial infarction. J Am Coll Cardiol 2014 Jun 17;63(23):2569-78

6. Than M, Aldous S, Lord S J, et al. A 2-hour diagnostic protocol for possible cardiac chest pain in the emergency department: a randomized clinical trial. JAMA Intern Med 2014;174(1):51-8.

7. Rathod K S, Ward H, Farooqi F. Chest pain symptom scoring can improve the quality of referrals to Rapid Access Chest Pain Clinic. BMJ Qual Improv Report 2014;3:1 u203864.w1691 doi:10.1136/bmjquality.u203864.w1691

8. Byrne J. Introducing a chest pain pathway in the emergency department to improve quality of care for patients with possible cardiac chest pain. BMJ Qual Improv Report 2014;3: doi:10.1136/bmjquality.u204753.w2003

\section{Declaration of interests}

Nothing to declare.

\section{Acknowledgements}

Dr Paul Huggan (general medicine consultant, Waikato DHB). 